\title{
AMNESTIE BEI DOSTOEVSKIJ
}

\author{
Sebastian Kornmesser, Dresden, Deutschland, \\ sebastian.kornmesser@googlemail.com
}

DOI: 10.31902/fll.38.2021.10

Der Begriff Amnestie bedeutet Straferlass von einer Gruppe von Menschen, ohne jedoch die Schuld zu tilgen. Amnestien werden meist im Zusammenhang mit politischen Ereignissen wie zwischenstaatlichen Friedens-Amnestien, in Zeiten des politischen Wandels oder Kriegen erlassen. Dabei gilt jedoch immer ein Verzicht auf strafrechtliche Verfolgung, die im Volksmund als, Gnade vor Recht' bekannt ist. Amnestien können daher zum einen als menschlicher Akt der Gnade betrachtet werden, doch können sie zum anderen auch Probleme im Gerechtigkeitsempfinden der Bevölkerung hervorrufen, da Strafen unterschiedlich geahndet und Gleichheitsprinzipien erschüttert werden. Die Begriffe Strafe, Schuld und Unschuld sowie ihre Darstellung sind daher Grundlage für die Frage, wie sich Amnestien in Dostoevskijs Texten herausbilden.

Dostoevskijs Figuren sind meist ambivalent und fordern eine reflektierte Lektüre wie auch ethische Grenzziehung heraus. Indem der Autor in seinen Texten die soziale Ungerechtigkeit zum Thema macht, gerät die Wiederherstellung der Gerechtigkeit durch die Amnestie in seinen Blick. Eine Mitverantwortung in der Gesellschaft sowie eine Mitschuld im höheren Sinne bilden dabei das Zentrum seiner Argumentation, zum einen als Kontrast zur Justiz, welche die Schuld des Einzelnen betrachtet, und zum anderen als Betrachtung der Erbschuld des Menschen und seine Verantwortung für die Gemeinde. In einem Vergleich zur antiken griechischen Rechtsprechung zeigt sich zudem, dass Amnestien ursprünglich im engen Zusammenhang mit Kollektiv, Emotion und Ritual standen. Daraus ergeben sich neue Betrachtungsweisen prominenter Texte Dostoevskijs, wie anhand von Brat'ja Karamazovy und anderer Werke gezeigt werden soll. Dadurch soll ein kurzer Überblick darüber gegeben werden, was Dostoevskij als Schuld und Unschuld versteht, welche Funktion Strafe in seinen Texten hat und wie sich dadurch Amnestie herausbildet.

Schlüsselwörter: Amnestie, Mitschuld, Unschuld, Schuld, Strafe, antike Rechtsprechung, Justiz, Gnade.

Anlässlich des 20. Jahrestages der russischen Verfassung am 12. Dezember 2013 verabschiedete die Duma ein Amnestiegesetz, mit dem die Entlassung von 25000 Inhaftierten angekündigt wurde. Michail Chodorkovskijs während seiner Haft geschriebene Textsammlung 
Tjuremnye ljudi (Meine Mitgefangenen) kommentiert im letzten Kapitel „Amnestia“ die Aussicht der Inhaftierten auf ihre Entlassung:

Amnestie ist wie das Hoffen auf ein Wunder. Und plötzlich ist es da. Man hält den Gesetzesentwurf in seinen Händen. Begierig durchsucht man ihn darauf, ob die eigenen Vergehen auch darunterfallen. Gefunden - großes Glück. Eine Hoffnung, die plötzlich Gestalt anzunehmen beginnt. Telefonate mit den Angehörigen, ihre gemeinsame angespannte Freude. Warten.

Nur die Altinsassen, in deren Leben kein Platz mehr ist für Illusionen, begegnen den Fragen mit einem skeptischen Lächeln. Man geht innen vorsichtig aus dem Weg. Ihre Skepsis macht einem Angst, ärgert einen sogar: Wie kann man nur nicht an das Wunder glauben? (Chodorkowski $90 \mathrm{f.}$ )

Im Gegensatz zu den neu Inhaftierten reagieren die Altinsassen mit Hoffnungslosigkeit und Resignation auf die allgemein hohen Erwartungen (90). Chodorkovskijs Text lässt offen, inwiefern die Amnestie tatsächlich zu einer Entlassung seiner Mitgefangenen wie auch ihm selbst geführt hat. ${ }^{1}$ Die Portraits seiner Mitgefangenen beschreiben insgesamt ein Bild von Inhaftierten, die sich trotz grausamer Haftbedingungen ihre ,Selbstachtung' bewahrt haben (11). Mit dem Begriff Amnestie übt Chodorkovskij Kritik am russischen Strafsystem, da sich in ihm die Willkür in der Bestrafung wie auch die Forderung nach Begnadigung des Menschen ausdrücke. Gleichzeitig verschleiert Tjuremnye ljudi aber mit dem Fokus auf die Menschlichkeit der Inhaftierten, zu denen er selbst gehörte, die Frage nach deren Schuld. Der Autor rechtfertigt sich in dieser Weise vor dem Leser - denn Amnestie bedeutet Straferlass, ohne jedoch die Schuld zu tilgen.

Diese (literarische) Rechtfertigung vor dem Hintergrund der eigenen Inhaftierung, die Betrachtung des grausamen Gefängnisalltages sowie der Menschlichkeit seiner Insassen in Tjuremnye ljudi knüpfen an die Tradition der sogenannten Lagerliteratur an, die ihren Anfang 1862 mit Dostoevskijs Zapiski iz mërtvogo doma (Aufzeichnungen aus dem

\footnotetext{
${ }^{1}$ Die Zahl der durch das Amnestiegesetz entlassenen Inhaftierten belief sich auf weit weniger als die angekündigten 25000. Chodorkovskij wurde am 25. Oktober 2003 verhaftet und wegen angeblicher Unterschlagung und Steuerhinterziehung verurteilt. Man entließ ihn schließlich, unabhängig vom Amnestiegesetz, nach einem eingereichten Gnadengesuch am 20. Dezember 2013. Während der letzten drei Jahre seiner Inhaftierung schrieb Chodorkovskij Portraits seiner Mitgefangenen, die als Kolumne u.a. in der Oppositionszeitschrift Novoe vremja veröffentlicht wurden und 2014 unter dem Titel Meine Mitgefangenen in Deutschland erschienen.
} 
Totenhaus) nehmen. ${ }^{2}$ So werden die Mitgefangenen bei Dostoevskij als Unschuldige und Kinder dargestellt, die in ihrer Verbannung in die sibirische Katorga in ähnlicher Weise auf ein Wunder hoffen dürfen. ${ }^{3} \mathrm{Im}$ Unterschied zu Tjuremnye ljudi wird in den Zapiski wie auch in anderen Werken Dostoevskijs der Begriff Amnestie jedoch nicht verwendet. Durch den einleitenden Satz aus dem Artikel Sreda (Das Milieu), „Betreten wir den Gerichtssaal mit dem Gedanken, daß wir mitschuldig sind" (a.a.O.), wird stattdessen an eine kollektive Mitschuld und Mitverantwortung appelliert, aus der heraus sich bei Dostoevskij Amnestie entwickelt. Auch in Brat'ja Karamazovy (Die Brüder Karamasov) findet sich ein ähnlicher Bezug zur Mitverantwortung, die mit der Maxime ,Jeder ist für alles verantwortlich' eine zentrale Rolle in der Textkomposition einnimmt. Daher stellt sich die Frage, inwiefern sich in Dostoevskijs Werken ein kollektiver Straferlass im Sinne einer Amnestie zeigt. Ein Vergleich zur antiken griechischen Rechtsprechung offenbart zudem, dass Amnestien bei Dostoevskij in einem Zusammenhang mit Kollektiv, Emotion und Ritual stehen. Dies soll anhand von Beispielen u.a. aus den Werken Zapiski iz mërtvogo doma, Dnevnik pisatel'ja und Brat'ja Karamazovy untersucht werden, um

${ }^{2}$ Dostoevskij führt dem Leser die Grausamkeiten der Inhaftierungszustände in einer bis dato unbekannt realistischen Weise vor Augen, die maßgebend für die nachfolgende sogenannte Lagerliteratur sein sollte. Mit der Darstellung des Lagers in den Zapiski knüpft Dostoevskij erfolgreich an die Gefängnisliteratur von Autoren wie z.B. Miguel de Cervantes an, der durch seine Erfahrungen als Galeerensklave die Inspiration für seinen Epoche machenden Roman Don Quijote (1605) hatte, oder John Bunyan, der The Pilgrim's Progress (1678) teilweise im Gefängnis schrieb und der die conversio eines Pilgers beschreibt wie auch De Sades während seiner Haft geschriebenen Werke wie Les centvingt jours de Sodome (1782) und Aline et Valcour ou Le Roman philosophique (1786). Vor allem Le dernier jour d'un condamné (1829) von Hugo spielt für Dostoevskij im Hinblick auf seine eigene Erfahrung als Delinquent wie auch für seine Erzähltechnik eine entscheidende Rolle. Hugo schildert die Erinnerung eines zum Tode verurteilten Inhaftierten, der in den letzten Wochen seines Lebens bis zum Tag seiner Hinrichtung von seinen Erinnerungen berichtet.

3 Dostoevskij wurde nach einer Scheinhinrichtung zu vier Jahren in der sibirischen Katorga verurteilt, was sein literarisches Schaffen ab den 1860er Jahren nachhaltig veränderte. Andrea Zink weist auf den ,Entlastungsmechanismus' der Zapiski hin, mit dem sich Dostoevskij selbst von jeder Schuld habe freischreiben wollen (Zink 133). Die Zusammenhänge zwischen Strafe und Tat in Dostoevskijs Darstellung der Inhaftierten und ihres Alltags seien weitestgehend ausgeblendet, so Zink (117), was so auch für Chodorkovskij Text gilt. In der Gemeinschaft der Inhaftierten gilt es, nicht darüber zu sprechen, warum jemand verurteilt worden sei. 
dadurch einen Verlauf in der Herausbildung der Amnestie bei Dostoevskij ab den 1860er Jahren nachzuzeichnen. Insgesamt soll ein kurzer Überblick darüber gegeben werden, was Dostoevskij als Schuld und Unschuld versteht, welche Funktion Strafe in seinen Texten hat und wie er Amnestie narrativiert.

\section{Unschuld, Schuld und Strafe}

Die Darstellung von Unschuld in Form einer Figur ist in Dostoevskijs Texten im Wesentlichen auf das Kindliche beschränkt und eingebettet in eine ambivalente narrative Struktur, die es zu deuten gilt. Der Protagonist Fürst Muškin aus Idiot (Der ldiot), ein naiver und kindlicher Gutmensch, scheint ein gutes Beispiel für die Darstellung einer unschuldigen Figur zu sein, doch wie Horst-Jürgen Gerigk zum Ausdruck bringt, ist die Unschuld der Figur ein Spiel mit der Erwartungshaltung des Lesers:

In poetologischer Hinsicht profiliert Dostoevskij in diesem Roman seinen Titelhelden objektiv als eine Person, die durch sittliche Reinheit definiert ist. [...] Der Erzähler schildert hier „von außen“ (mit ganz wenigen Ausnahmen), wodurch wir keine Direktaufnahme des Innenlebens der Titelfigur erhalten. Wir können nicht wissen, was in Myschkin vorgeht. Deshalb werden wir in dem Moment, als Myschkin das Schreiben des Rechtsanwalts Salaskin hervorzaubert, das sein Millionenerbe bescheinigt, zur Frage gedrängt, ob wir denn diesen Fürsten bislang verkannt haben, als wir ihn als sympathischen und naiven jungen Mann (26 Jahre alt) einschätzten, der sein Herz auf der Zunge trägt. (Gerigk/Neuhäuser 27 f.)

Nach Gerigk wird die Darstellung von Unschuld bei Idiot durch das narrative Mittel der auktorialen Erzählweise verschleiert. Myškins Untaten überraschen uns und provozieren damit die Frage, inwiefern Protagonisten gänzlich unschuldig sein können. Die Verschleierung von Unschuld und Schuld durch narrative Mittel lässt sich auch an Dostoevskijs unzuverlässigen Erzählerstimmen aufzeigen, mit der die Glaubwürdigkeit des Erzählens insgesamt in Frage gestellt wird. So ist der Erzähler in Besy (Die bösen Geister) selbst Teil der Verbrechensvorgänge innerhalb der auf Lügen und Denunziationen basierende Welt der bösen Geister, oder wir lauschen zusammen mit dem Protagonisten und Erzähler Gorjančikov in den Zapiski der Eifersuchtstragödie um Akulkas Mann. Durch diese „Hölle der Eifersucht in einer Mischung aus Scham, Arroganz und entfesseltem Sadismus", so Gerigk, (Gerigk/Neuhäuser 23 f.), werden die Untaten Gorjančikovs erst 
greifbar gemacht, indem der Leser durch diese Parallelgeschichte „Einblick in die verschlossene Seele des Erzählers [erhält], der über alles Mögliche berichtet, nur nicht über sein eigenes Verbrechen. Erst eine reflektierte Lektüre läßt uns diesen Kunstgriff Dostoevskijs wahrnehmen." $(23)^{4}$

Die narrative Perspektive ist demnach entscheidend für die Beurteilung der Unschuld bzw. Schuld einer Figur und provoziert durch die Ambivalenz gleichzeitig eine ethische Grenzüberschreitung. Laut Dagmar Burkhart gehe es bei Dostoevskijs Protagonisten stets um die ,Notwendigkeit einer Grenzziehung', da das Fehlen von Grenzen zur ,existenzbedrohenden Orientierungslosigkeit' führe (Burkhart 48). Dostoevskij vertrete dabei eine „Verantwortungsethik", die das sittliche Handeln als Pflicht voraussetze und die er mit einer christlichen Vorstellung von Moral verbinde: Die Protagonisten seien entweder Repräsentanten dieser moralischen Grundsätze, oder sie setzen sich durch das Verbrechen über deren Grenzen hinweg (48). Gänzliche Unschuld in den Figuren Dostoevskijs sucht man daher meist vergeblich. Stattdessen verschwimmen Unschuld und Schuld miteinander und bedingen sich gegenseitig.

Diese auf die alttestamentarische Erbschuld zurückzuführende Wertehaltung einer Schuldigkeit aller Menschen vor Gott ist Grundlage der von Dostoevskij postulierten Mitschuld der Menschen an weltlichen Verbrechen, die sie nicht unmittelbar selbst ausgeführt haben müssen, aber für dessen Entstehung sie eine Mitverantwortung innerhalb der Gemeinschaft tragen. Die Begriffe Schuld, Mitschuld und Unschuld stehen dabei insgesamt im engen Kontext zur biblischen Betrachtungsweise einer Rechtsprechung mit Gott als Richter, während die juridische Betrachtungsweise weltlicher Rechtsprechung in Dostoevskijs Texten meist mit Begriffen wie Strafe, Justiz und Urteil beschrieben werden. Da sich die weltliche Gerichtsbarkeit bei Dostoevskij meist als unzureichend und nicht zielführend herausstellt

4 Neuhäuser macht ebenfalls auf die Problematik der perspektivischen Konstruktion aufmerksam anhand des Beispiels Prestuplenie $i$ nakazanie (Schuld und Sühne). Seiner Meinung nach liege sie in der Entstehungsgeschichte des Werkes begründet, da Prestuplenie i nakazanie zuerst als Ich-Erzählung, als ,Bericht des Mörders' konzipiert worden sei und erst später mit der Einbeziehung eines, allwissenden Autors' eine Veränderung durch den Autor erfuhr (Neuhäuser 179). Dostoevskij empfand die Erkenntnisfunktion des Subjekts als zu eng konzipiert. Diese ,quasi-objektive Erzählperspektive', in der sich der Leser „zugleich innerhalb und außerhalb des Bewusstseins des Helden“ befinde, so Neuhäuser, sei paradigmatisch für die Sprache und den Stil des gesamten Textes (179). 
(vgl. den Justizirrtum bei Brat'ja Karamazovy oder das Strafsystem der Katorga in den Zapiski), zeigen sich die Figuren stattdessen als schuldig vor Gott.

Die Thematisierung von Schuld hingegen findet man bei Dostoevskij in solch einer Vielfalt wie bei kaum einem anderen Autor seiner Zeit: Fëdor Karamazov und seine Söhne, der Erzähler der Zapiski oder von Krotkaja (Die Sanfte), Raskolnikov im Roman Prestuplenie $i$ nakazanie (Schuld und Sühne), Pëtr Verchovenskijs in Besy (Die Dämonen); sie alle haben sich schuldig gemacht durch Mord, Verleumdung oder Vergewaltigung, doch bleibt ihre Darstellung ähnlich ambivalent wie die der Unschuld. Diese von Gerigk als ,machiavellistische Poetik' bezeichnete Aufwertung und Polarisierung der mit Schuld beladenen Figuren innerhalb der Themenbereiche Verbrechen, Krankheit, Sexualität und Religion in Dostoevskijs Texten richte sich, so Gerigk weiter, an den Geschmack des ,sensationslüsternen' Massenpublikums von Zeitschriften, in denen Dostoevskij seine Texte veröffentlichte (Gerigk 160). Doch Schuld bietet in narrativer Hinsicht einen größeren Spannungsbogen und größere Möglichkeit für den Autor, seine Figuren und ihr Schicksal als Neubeginn und Sinneswandel zu inszenieren.

Strafe, im Unterschied zur Schuld, ist meist mit der Darstellung von Körperstrafen verknüpft, mit denen die Ungerechtigkeit insbesondere von Haftstrafen angemahnt werden. So werden in den Zapiski die Grausamkeiten des sadistischen Lageraufsehers Šerebjatnikov und seiner Körperstrafen in aller (realistischer) Detailschärfe geschildert, um die Grausamkeit des Strafsystems als Folge ungerechter Verurteilung anzuklagen:

Am meisten jedoch, erinnere ich mich, beschäftigte mich ein Gedanke, der mich fortan während meines Gefängnisdaseins verfolgte und für mich ein zum Teil unlösbares Problem darstellte, das ich auch heute noch nicht zu lösen vermag: die ungleiche Bestrafung für gleiche Verbrechen. Ein Verbrechen läßt sich fürwahr nicht mit einem andern vergleichen, auch nicht annähernd. Ein Beispiel: da sind zwei Menschen, die beide jemand ermordet haben. Alle Umstände der beiden Taten werden abgewogen; und für die eine wie für die andere ergeht fast das gleiche Urteil. Dabei halte man sich einmal vor Augen, welch ein Unterschied zwischen den Verbrechen besteht! (Dostojewski 1983, 67)

Diese Gegenüberstellung zweier ungleicher Verbrechen, die jedoch gleich bestraft werden, verweist auf das Gleichnis von Jakob und Esau 
bzw. auf die biblische Frage nach der Gerechtigkeit Gottes bei der ungleichen Bestrafung zweier gleicher Sünder. In Augustinus' Lehre nimmt die Argumentation für die Gerechtigkeit Gottes anhand dieses Beispiels eine zentrale Rolle in seiner Gnadenlehre ein. Bei Augustinus wird die Bestrafung Esaus und die Gnade, die Jakob zuteilwird, obwohl beide als Zwillingsbrüder gleich vor Gott sind, mit der Erbsünde Adams erklärt. Der Mensch als dessen Nachkomme trage die Sünde in sich und müsse sich in Folge dessen auch einer gerechten Strafe unterziehen. Wenn aber Gott, wie im Falle von Jakob, nicht straft, sondern Gnade walten lässt, so zeugt dies von der Gerechtigkeit Gottes und von seiner Größe (Drecoll, 488ff.).

Augustinus' Gnadenlehre und Theorie der Erbsünde, die seine Rezipienten vor allem im Mittelalter zur Rechtfertigung von Ungerechtigkeit und Bestrafung seitens der Kirche (aus)nutzten, wird in den Zapiski zur Darstellung der babylonischen Welt der Katorga, die nur in spiritueller Hinsicht für den Protagonisten ein Ausweg in die Freiheit, in ein Leben in pace bereithält. Im Unterschied zu Augustinus zeigt sich bei Dostoevskij die gleiche Bestrafung für ungleiche Verbrechen lediglich als eine Bestrafungslehre, die zwar ebenfalls von der Sünde des Menschen bzw. von seiner Schuld vor dem Gesetz ausgeht, die jedoch keine Option zur Begnadigung bereithält: Das Lagergesetz kann nicht begnadigen, sondern muss die Bestrafung innerhalb des Strafsystems aufrechterhalten. Ähnlich wie Augustinus so zeigt auch Dostoevskij, dass Gnade wie die Hoffnung auf ein Wunder ist, welches nur wenigen zuteilwird, und dies nur im Sinneswandel und Bekenntnis zu Gott. ${ }^{5}$

Schuld und Strafe innerhalb der ambivalenten Darstellung der Figuren bei Dostoevskij zielen insgesamt auf eine mögliche Erlösung als Neubeginn und Sinneswandel ab, der jedoch nicht auf juridischem, sondern auf christlichem Boden gedeihen soll, wie es die einleitenden Zeilen der Brat'ja Karamazovy ankündigen: Wahrlich, wahrlich, ich sage euch: Wenn das Weizenkorn nicht in die Erde fällt und erstirbt, bleibt es allein; wenn es aber erstirbt, bringt es viel Frucht. (NT Joh 12,24). Die Strafe dient dabei als Ventil für ein Bekenntnis zur Schuld (im Sinne der Erbschuld). Bekenntnisse bzw. Beichten können so zu einer Lösung aus

\footnotetext{
${ }^{5}$ Ein Vergleich von Dostoevskijs Zapiski zu Augustinus De Confessiones zeigt einen ähnlichen Zugang zur Rechtfertigung des Glaubens vor dem Hintergrund der Bekehrung und Auseinandersetzung mit der Glaubwürdigkeit literarischer Argumentation. Augustinus geht es nicht zuletzt um die Rechtfertigung des eigenen Schreibens seines Glaubensbekenntnisses, d.h., das Glaubhaft-Machen der eigenen Bekehrung vor der Leserschaft.
} 
dem Bestrafungssystem führen, was die zahlreichen Bekenntnisse in Dostoevskijs Texten belegen, doch sind sie kein Garant für die Erlösung, so wie ,das Hoffen auf ein Wunder' (a.a.O.) nur Hoffen bleiben kann. Bekenntnisse zeigen, wie wichtig der damit einhergehende Sinneswandel ist und dass dieser authentisch und nicht inszeniert sein darf, wie in Stavrogins schriftlicher Beichte im Beisein des Mönches Tichon in Besy. Das Bekenntnis (zu Gott) sowie die Beichte (der eigenen Sünden) bedeuten bei Dostoevskij, dass der Einzelne sich seiner (Erb)Schuld bewusst wird und damit in eine Gemeinschaft derer eintritt, die mit ihrem Bekenntnis Gleiches tun. Dies führt zu einer Betrachtung der Gemeinschaft vor dem Hintergrund einer kollektiven Schuld bzw. der mit Amnestie in Verbindung stehenden Mitschuld.

\section{Mitschuld und Amnestie}

Der Begriff Amnestie wird heutzutage meist in Verbindung mit einem politisch motivierten Straferlass einer Gruppe von Menschen wie zwischenstaatlichen Friedens-Amnestien assoziiert oder ausgelöst durch Zeiten des politischen Wandels oder des Krieges. Dabei gilt jedoch immer ein Verzicht auf strafrechtliche Verfolgung, die im Volksmund als ,Gnade vor Recht' bekannt ist. Amnestien können daher zum einen als menschlicher Akt der Gnade betrachtet werden, doch können sie zum anderen auch Probleme im Gerechtigkeitsempfinden der Bevölkerung hervorrufen, da Strafen unterschiedlich geahndet und Gleichheitsprinzipien erschüttert werden. Doch gerade die Betrachtung der Amnestie als Wiederherstellung der sozialen Gerechtigkeit macht das Konzept Dostoevskijs von Mitschuld und Mitverantwortung so aktuell. Seine zugrundeliegende politische Haltung für dieses Konzept basiert auf den Ideen der počvenniki, zu denen er sich selbst zählte und die er mit der Herausgabe seiner Zeitschriften Vremja und Épocha ab den 1860er Jahren Ausdruck verlieh. So führt Joseph Frank die politischphilosophische Haltung der Vremja und Épocha auf eine friedfertige Utopie der počvenničestvo (Rückkehr zum Erdboden) zurück, die eine weitestgehend politisch angepasste und diplomatische Ausrichtung vertreten habe (Frank 2002, 36 f.):

The pochvenniki, in other words, believed that the social-political issues of the day should be seen as secondary to the larger task of helping to forward a new Russian cultural synthesis - one that would emerge from the fusion of the people and their more cultivated superiors. For the radical intelligentsia, exactly the reverse hierarchy of values prevailed: all other issues were secondary to that of improving the lot of the peasantry in the manner they considered most consonant with social justice. 
Another noticeable feature of Dostoevsky's program, which again sets it off sharply from that of the radicals, is his insistence that the transformation must take place peacefully and his conviction (or hope) that violence would be avoided. (Frank 2002, 35)

Dieses Programm eines friedvollen Wandels in der Gesellschaft in Vremja und Épocha, so Frank weiter, distanziere sich damit sowohl von den konservativen wie auch von liberalen Denkern, zu denen zu diesem Zeitpunkt Teile der Slavophilen und die als radikal empfundene Position der Zeitschrift Sovremmenik (Der Zeitgenosse) gezählt wurden (36). Entscheidend für die počvenniki war die kulturelle Synthese unterschiedlicher Volksschichten, welche den Abbau von Hierarchien und die Aufwertung des bäuerlichen Lebens ermöglichen sollten, um für die Frage nach der sozialen Gerechtigkeit Verantwortung zu tragen. (So wurde erst 20 Jahre vor dem Erscheinen von Dostoevskijs Zeitschriften Vremja und Épocha die Leibeigenschaft in Russland abgeschafft.) Die počvenniki sehen also einerseits einer friedvollen politischen Neuerung entgegen, die erst im Kollektiv entsteht. Andererseits versuchen sie, dem revolutionären Geist dieser neuen liberalen Epoche der 1860er Jahre Ausdruck zu verleihen, indem sie vor allem Intellektuelle und Literaten zu Wort kommen lassen.

Diese Haltung der počvenniki zeigt sich bei Dostoevskij erstmals in den in Vremja veröffentlichten Zapiski, insbesondere im Verhältnis zwischen dem Protagonisten Gorjančikov und seinen Mitgefangenen. Aleksandr Petrovič Gorjančikov begeht einen Mord an seiner Ehefrau und landet dafür in der sibirischen Katorga. Nach dem Ende seiner Haft, welche unter grausamsten Bedingungen stattfindet, legt er mit dem Lagerbericht ein Zeugnis über die Haftbedingungen sowie über seinen Sinneswandel während der Haft ab. Gorjančikov wird als jemand beschrieben, der aus adliger Herkunft stammt und während seiner Haft von den anderen Inhaftierten anfänglich weitestgehend isoliert bleibt. Der exemplarisch durch den Protagonisten Gorjančikov zum Ausdruck gebrachte Sinneswandel des Volkes, an dessen Beispiel sich auch Dostoevskijs eigener Sinneswandel ablesen lässt, versucht das Einzelschicksal eines Inhaftierten mit dem Kollektiv aller Inhaftierten, dem Volk, zu synthetisieren. ${ }^{6}$ Dostoevskij spreche für sich selbst, so

${ }^{6}$ Durch die autobiographische Erzählweise der Zapiski ist der biographische Gehalt des Werkes in der literaturwissenschaftlichen Forschung oft debattiert worden; so macht z.B. Frank auf die ambivalente Haltung Dostoevskijs aufmerksam, der einerseits die Identifikation mit dem Protagonisten möglichst verdecken möchte, dabei aber gleichzeitig auf seinen Namen als Autor 
Andrea Zink, wenn er mit dem Protagonisten Gorjančikov eine ,gesellschaftliche Elite' schildere, ,die sich durch das Volk vor dem Volk' retten will, (Zink 133). Doch entgegen eines bloßen Selbstzweckes für den Adel postuliert Dostoevskij stattdessen mit dem Werk eine Annäherung von Volk und Adel im Sinne des friedvollen Wandels der počvenniki. Nach Frank hieße dies, dass eine kulturelle Synthese von Volk und intelligencija angestrebt wird, indem Hierarchien abgebaut und soziale Gerechtigkeit wiederhergestellt werden.

Gorjančikov ist schuldig und die grausamen Haftbedingungen zeigen keine Rehabilitierung durch die Haft. Doch die Nähe zu seinen Mitgefangenen rehabilitiert ihn zunehmend von der Entfremdung zum Volk und führt damit zu einer Versöhnung beider Gruppierungen. Darin zeigt sich mit den Zapiski erstmals eine Annäherung Dostoevskijs an die Herausbildung der Amnestie, als Wiederherstellung der sozialen Gerechtigkeit durch die Annäherung und Versöhnung von Volk und Adel. Die Versöhnung selbst bleibt jedoch unausgesprochen und wird im Text nur angedeutet. Dass sich die Inhaftierten ihrer eigenen Schuld zudem nicht bewusst sind, macht eine Forderung nach Begnadigung der Inhaftierten im Sinne eines Straferlasses, ohne die Schuld zu tilgen, zumindest fragwürdig. Damit wird eine gewisse Skepsis sichtbar, mit der Dostoevskij in den frühen 1860er Jahren die Umformung der Gesellschaft durch die Synthese des russischen Volkes betrachtete. Erst in den kommenden Jahren wird diese Skepsis einer zunehmend religiösen Betrachtungsweise der Amnestie weichen.

Gut zehn Jahre nach den Zapiski veröffentlicht Dostoevskij 1873 den Artikel Sreda im Dnevnik pisatel'ja (Tagebuch eines Schriftstellers) und mahnt die Mitschuld von Geschworenen sowie die Ungerechtigkeit eines strafrechtlichen Prozesses an. Der Artikel beschäftigt sich in erster Linie mit dem durch die Justizreform von 1864 neu eingeführten Geschworenengericht und den daraus resultierenden übermäßigen Freisprüchen von Angeklagten. Wichtig an diesem Prozess sind für Dostoevskij vor allem der Aspekt der Zerstörung der kindlichen Unschuld sowie ein Verbrechen an der Mutter des Kindes vor dem Hintergrund einer juristischen Fehlentscheidung. ${ }^{7}$

aufmerksam machen will (Frank 1986, 214). Laut Frank zeigen Dostoevskijs Zapiski vor allem in Anlehnung an Victor Hugos Le dernier jour d'un condamné einen ähnlichen Zugang zu christlichen Themen und seien damit richtungsweisend für Dostoevskijs späteres Schaffen bis hin zu Brat'ja Karamazovy (198.).

7 Dostoevskijs großes Interesse am Geschworenengericht, insbesondere in Verbindung mit der Verurteilung von Kindern, geht zurück auf den Prozess des 
Der Artikel Sreda beginnt mit der Frage, warum das in Russland 1864 durch die Justizreform neu eingeführte Geschworenengericht einen Ehemann freispricht, der zuvor mehrfach seine Frau verprügelt hat, bis diese sich erhängt, um den Schlägen ihres Mannes zu entgehen. Die grausamen Taten des Ehemanns an seiner Frau (während das Kind zuschaut) werden in Sreda detailliert nacherzählt. Das Bild der gequälten und unschuldigen Frau sowie ihres Kindes soll nicht zuletzt an das Mitleid der Leserschaft appellieren, stellvertretend im Text durch die Geschworenen. Dostoevskij spitzt im weiteren Verlauf des Artikels die Erklärung für den Freispruch des Mannes auf das Milieu und die Selbstbezichtigung der Geschworenen zu:

'Also soll man ihn eben deshalb freisprechen?'

Nein, im Gegenteil: eben deshalb muß man die Wahrheit sagen und das Böse, ohne Scheu das Böse nennen, dafür aber die Hälfte der Bürde des Urteils auf sich nehmen. Betreten wir den Gerichtssaal mit dem Gedanken, daß wir mitschuldig sind! Dieser Schmerz im Herzen, den jetzt alle so sehr fürchten und mit dem wir den Gerichtssaal verlassen, wird dann unsere Strafe sein. Denn es ist doch so, daß wir, indem wir selbst besser werden, auch das Milieu verbessern. Und nur auf diese Weise ist das doch $\mathrm{zu}$ bewerkstelligen. (Dostojewski 30)

Nach der Logik dieser Aussage, die Dostoevskij in das komplizierte Geflecht polyphoner Stimmen im Text einbettet, nehmen die Geschworenen mit der „Hälfte der Bürde des Urteils“ auch die Hälfte des Verbrechens des Angeklagten auf sich, was zu einem schwierigen Verhältnis der Geschworenen innerhalb der Rechtsprechung führt. Der Appell an die Mitschuld geht im Verlauf des Artikels sogar so weit, dass nach der Vorgehensweise der Geschworenen, nahezu alle freizusprechen, das Verbrechen zu einer Pflicht würde, zu einem ,edlen Protest gegen das Milieu' (ebd.). Dostoevskij bringt schließlich das ,Christentum' und eine, sittliche Pflicht' als Gegenargument ins Feld, welche sich gegenüber dieser Grausamkeit und einer ,Lehre des Milieus' behaupten müsse (31).

Der Artikel endet damit, dass die Advokatur und ihre irreführenden rhetorischen Reden für diesen Justizirrtum verantwortlich gemacht

Jahres 1867 um Ol'ga Umeckaja. Dostoevskij schreibt in Sreda aus aktuellem Anlass und zum ersten Mal über Gerichtsfälle in Russland und greift damit u.a. auf seine seit dem Umeckaja-Prozess angesammelten Recherchen zurück (Rosenshield 36). Dostoevskijs großes Interesse an diesen Fällen lässt sich bis hin zu Brat'ja Karamazovy verfolgen. 
werden. Diese Umkehrung der Positionen von Kläger und Angeklagtem, von Opfer und Täter, stellt einen wichtigen Teil der Strategie dar, mit der Dostoevskij gegen die Justiz sowie gegen den Advokatenstand polemisiert. Mitschuld bedeutet in dieser religiösen Betrachtungsweise, die Versündigung an christlichen Wertvorstellungen des Zusammenlebens. In anderer Stelle in Sreda wird versucht, die übermäßigen Freisprüche aus jenen christlichen Werten heraus als Akt der Gnade zu erklären:

Diese schreckliche Macht über das Menschenschicksal, über das Schicksal unserer leiblichen Brüder hat uns erschreckt, und bevor wir zu unserem Staatsbürgertum heranreifen, üben wir Gnade. Aus Angst üben wir Gnade. Wir sitzen als Geschworene da und denken vielleicht: ,Sind wir denn selbst besser als der Angeklagte? Wir sind reich, versorgt, sollte es aber geschehen, daß wir in dieselbe Lage gerieten wie er, so würden wir vielleicht noch Schlimmeres tun als er. Darum üben wir Gnade.' So könnte es sich denn vielleicht noch herausstellen, daß dieses Ergriffensein des Herzens, das uns abhält, zu verurteilen, etwas Gutes ist. Vielleicht ist es ein Unterpfand für irgendetwas höher Christliches in der Zukunft, wovon die Welt bisher noch nichts weiß! (Dostojewski 29)

In gewisser Weise ließe sich der übermäßige Freispruch der Angeklagten selbst als Amnestie bezeichnen, eine Amnestie des Volkes als ,edler Protest gegen das Milieu' (a.a.O.), die jedoch aus Angst und eigener Unzulänglichkeit heraus freispricht. Auch wenn Dostoevskij die Freisprüche anmahnt, da sie die offensichtliche Schuld des angeklagten Ehemannes ungeahndet lässt, stellt sich hier der Akt der Gnade als positive russische Eigenart heraus. Auf der Grundlage der Mitschuld liegt in der Gnade das Potential eines zukünftigen und höheren Christlichem, das im Sinne einer religiösen Amnestie erst in Brat'ja Karamazovy narrativ umgesetzt wird. Mitschuld und Gnade zeigen sich jedoch schon in Sreda als die entscheidenden kollektivgeprägten Unterschiede zur kulturgeprägten Formalität und Rationalität westlicher Moral- und Rechtsvorstellungen, nach denen die Justizreform von 1864 durchgeführt worden ist und die zu erheblichen Problemen in der Rechtsprechung geführt haben.

Diese religiöse Betrachtung von kollektiver Mitschuld bei Dostoevskij lässt sich am Beispiel der griechischen Geschichte der Amnestie seit der Antike in einen Zusammenhang zur Emotion und Rechtsprechung bringen. So beschreibt Angelos Chaniotis Amnestien als Kontrollmechanismen von Emotion und Erinnerung: 
[Die Amnestie] stellt einen rechtlichen Eingriff in emotionale und kognitive Prozesse dar, in Erinnerung und Emotion. Vergessen und Vergeben ist die Amnestie nicht, sondern Verbot und Verzicht: Verbot des Zornes und Verzicht auf die aus Zorn, Ehrgefühl und Rechtsempfinden resultierenden Handlungen der Bestrafung und Rache. (Chaniotis 50)

Zunächst lässt sich festhalten, dass eine unmittelbare Nähe des Begriffes Amnestie zur Amnesie ( $\alpha \mu \nu \eta \sigma i \alpha)$ besteht. Der seit dem 17. Jahrhundert in Russland verwendete Begriff Amnestie hat seinen Ursprung im altgriechischen á $\mu v \eta \sigma \tau i \alpha$ und bezieht sich auf ,Vergessenheit und Verzeihung eines Unrechts' (Vasmer 17) ${ }^{8}$ Chaniotis beschreibt Amnestien als einen, rechtlichen Eingriff' in das Erinnern an Untaten (Chaniotis 51). In Abgrenzung zu der im alten Griechenland so wichtigen Bedeutung der Rache und des Zorns als Vorzug vor Versöhnung, die sich auch literarisch in den Ilias wiederfindet, zeigt Chaniotis auf, wie wichtig die in der griechischen Rechtsprechung der Antike zunehmend praktizierte Amnestie für die Herausbildung der Eintracht in der Gemeinde gewesen sei. Doch müsse zunächst unterschieden werden zwischen dem "Verzicht auf eine rechtliche Verfolgung von Straftaten (Amnestie im eigentlichen Sinne) und dem Verzicht auf Rache und Vergeltung", so Chaniotis (48):

Wenn man auf die rechtliche Verfolgung von Straftätern und auf Rache verzichtet, vernachlässigt man zentrale Pflichten: die Pflicht, Recht zu sprechen und zu bestrafen, und die Pflicht, zu vergelten. Man tut dies zugunsten einer anderen Pflicht - der Pflicht, durch Versöhnung und Eintracht die Gemeinde zu retten. Was soll größeres Gewicht haben? Vergeltung (timorein) und Recht (dike) oder Eintracht (homonoia)? Das Recht der Toten oder der Vorteil der Lebenden? (61)

Die Frage, was größeres Gewicht haben soll, stellt sich auch Dostoevskij, indem er den Prozess (dike) um die drei Brüder Karamasow zwischen den beiden Maximen ,Jeder ist für alles verantwortlich'

\footnotetext{
${ }^{8}$ Vgl. dazu auch Harter-Uibopuu K./Mitthof F.: „Das moderne Recht versteht unter Amnestie den Verzicht eines Staates auf Strafverfolgung und Strafvollstreckung gegenüber einer Anzahl nicht individuell bestimmter Personen aus Gründen der Zweckmäßigkeit. Dieser Maßnahme stehen die Begnadigung, die den Strafverzicht gegenüber einer verurteilten Einzelperson bewirkt, und die Abolition, die Niederschlagung eines schwebenden Verfahrens, gegenüber." (8)
} 
(homonoia) und ,Alles ist erlaubt' (timorein) einpendelt. Auch in Brat'ja Karamazovy geht es um die Wiederherstellung von Eintracht und Harmonie in der dörflichen Gemeinde von "Viehhofen“ (Dostojewskij 2011, 912). In der Antike sei laut Chaniotis jedoch entscheidend für den Erfolg von Amnestien gewesen, sie mit Ritualen zu begleiten. Da durch Amnestien auch eine emotionale Kontrolle ausgeübt worden sei, indem das Gebot auf Rache zu verzichten ausgesprochen wurde, konnten Amnestien nur durch rituelle Handlungen gefestigt werden (Chaniotis 63). Die Amnestie sei dabei immer auf eine Erinnerung an ,rezente Ereignisse' gerichtet, die ,nicht älter als eine Generation' gewesen seien, was rituelle Handlungen umso wichtiger gemacht habe. ${ }^{9}$

Diese Betrachtung der Amnestie als Herausbildung einer Eintracht der Gemeinde, welche sich aus dem Geflecht von Erinnerung, Rechtsprechung und Ritual bildet, findet sich insbesondere im letzten Kapitel Reč' u kamnja (Die Rede an dem Stein) in Brat'ja Karamazovy wieder. Nach Koljas Frage, ob Dmitrij schuldig gesprochen wurde, antwortet Alëša, er sei unschuldig. Dann beginnt er seine Rede am Stein, mit der er alle Kinder anspricht, die um inn herum versammelt dem verstorbenen Kind Iljuša gedenken:

Hier, an Iljuschas Stein, wollen wir uns vornehmen, daß wir etwas Bestimmtes niemals vergessen - erstens, Iljuschetschka, und zweitens, einander. Und was auch immer das Leben uns später

${ }^{9}$ Chaniotis führt am Beispiel des Phrynichos zu den ,rezenten Ereignissen' aus: Die Episode um Phrynichos belegt eine mit rechtlichen Maßnahmen implementierte Kontrolle von Erinnerung, und zwar Erinnerung im engen und eigentlichen Sinne des Wortes. Es handelt sich um die Erinnerung an das gemeinsam Erlebte (also um, kollektives Gedächtnis') und nicht um ,Erinnerung' im übertragenen Sinne mit der Tradition des vor langer Zeit Geschehenen (also mit ,kulturellem Gedächtnis'). Der Unterschied ist signifikant. Die ältere Tradition wird oft, leicht und vielfach Objekt von Selektion, Gestaltung und Umformung. Der Kult der Vorfahren, die Pflege von historischen Denkmälern und Orten der Erinnerung, Hymnen und panegyrische Reden bei einem Fest, öffentliche Vorträge von Historikern im Gymnasium und im Theater, die Zerstörung von unerwünschten Zeugnissen der Vergangenheit (z.B. von Inschriften) - das alles sind einige der vielfältigen Medien, durch welche eine Gemeinde (und vor allem ihre Elite) das kulturelle Gedächtnis gestaltet und kontrolliert. Die deutlichste Institutionalisierung einer verordneten selektiven Erinnerung ist der historische Gedenktag. Die generelle Amnestie betrifft dagegen immer die Erinnerung an rezente Ereignisse, nicht älter als eine Generation. Die Kontrolle rezenter Erinnerungen ist natürlich wesentlich schwieriger, weil diese eine viel stärkere emotionale Dimension haben. (Chaniotis 53f.) 
bringen wird, sollten wir uns auch zwanzig Jahre nicht wiederbegegnen - wir wollen uns ewig daran erinnern, wie wir den armen Jungen beerdigten, den wir einmal mit Steinen beworfen haben - wissen Sie noch, an dem Steg? - und später so sehr ins Herz schlossen. (Dostojewskij 2011, 1236)

Am Tod Iljušas, der aus ärmlichen Verhältnissen kommt, haben sich gleich mehrere Personen schuldig gemacht: Zum einen trägt er Verletzungen von den Steinen davon, mit denen die anderen Kinder ihn beworfen haben, was von Alëša im letzten Moment unterbunden werden konnte; zum anderen sind es seine bäuerlichen Verhältnisse, in denen er aufwächst und durch die er zur Schwindsucht neigt sowie vor allem die Scham vor der Erniedrigung seines Vaters durch Dmitrij, die am meisten zu seinem Tod beigetragen hat. Daher ist die Frage nach Dmitrijs Schuld durch den Jungen Kolja kurz vor der Rede am Stein nicht zufällig und bereitet die Schuldfrage vor, welche auch für die Kinder selbst gilt: Bin ich schuldig vor Gott? Alëšas Antwort lautet ja und ist ein Appell an das kollektive Erinnern sowohl an die Tat des Steinewerfens, an der sie sich schuldig gemacht haben und an das sie Alëša mahnt (,wissen Sie noch, an dem Steg'), wie auch am Tod Iljušas, für den sie nach der Logik der Mitschuld ebenfalls Mitverantwortung tragen. Die Aussage, "sollten wir uns auch zwanzig Jahre nicht wiederbegegnen“ macht die Rede zudem zu einer einmaligen rituellen Handlung, die nicht im Sinne eines kulturellen Gedächtnisses jährlich bzw. regelmäßig widerholt wird, sondern auf „rezente Ereignisse“ (a.a.O.) reagiert.

Alëša hält seine Rede nicht etwa im Gericht oder im Haus der trauernden Eltern, sondern am Stein, den Iljuša besonders liebte. Dieser Stein wie auch das Pathos der Rede sowie das Publikum, welches nur aus Kindern besteht, wirken in ihrer ästhetischen Konzeption ganzheitlich und rituell. Die gesonderte Stellung der Rede als Epilog erhöht ihre Bedeutung über die gerichtlichen Reden und macht die Mitverschuldung bedeutungsvoller als die Schuld des Einzelnen. Vor Gott sind alle Beteiligten schuldig - sogar die Kinder. Doch durch die Verknüpfung von Untat und Erinnern innerhalb des Rituals der Rede am Stein ist es möglich, eine Amnestie als Verzicht auf rechtliche Strafverfolgung (die angesichts des Bauernjungen niemand unternehmen würde) zu gewähren, um die Gemeinde durch Versöhnung zusammenzubringen.

Es ist Alëša, der ihnen diese Amnestie gewährt und am Ende der Brat'ja Karamazovy mit folgenden Worten auf das einleitende Motto über das Samenkorn verweist: „Sogar, wenn nur eine einzige gute Erinnerung in unseren Herzen bleibt, kann sie irgendwann einmal 
unsere Rettung sein." (Dostojewskij 2011, 1239) Eben dieser Verweis macht seine Rede am Ende des Werkes zu einer religiösen Amnestie, welche die Hoffnung auf das Gute im Menschen, das dieser einmal gepflanzt hat, oder an das er sich durch eine gute Tat erinnert, als Verheißung auf dessen Schuldfreiheit und Gnade in Aussicht stellt. Jene Verheißung wird schließlich am Ende der Rede mit einem Hosianna der Kinder begleitet:

,O ja, o ja! Ewig, ewig!' riefen alle Jungen bewegt mit ihren hellen Stimmen. [...]

Ein Hurra auf Karamasow!' schmetterte Kolja begeistert.

,Und dem verstorbenen Jungen ewiges Andenken!' fügte Aljoscha aus tiefster Seele hinzu.

,Ewiges Angedenken!'stimmten die Jungen ein.

,Karamasow!' rief Kolja. ,Soll denn das wahr sein, was die Religion sagt, daß wir alle von den Toten auferstehen und wieder leben und uns wiedersehen werden, wir alle, auch Iljuschetschka?"

,Unbedingt werden wir auferstehen, unbedingt werden wir uns wiedersehen und heiter, freudig einander alles erzählen, was war' antwortete Aljoscha halb lachend, halb triumphierend. (1240)

Verheißung bedeutet hier Auferstehung und ein Leben nach dem Tod, in dem alles vergolten wird. Dieser Triumph, wie ihn Alëša und die Kinder empfinden, trägt sie über emotionale und kognitive Prozesse sowie über die Sphäre des irdischen Rechts hinaus im Sinne einer religiösen Amnestie, die eine Versöhnung im Jenseits für alle verheißt.

Amnestie zeigt sich bei Dostoevskij abschließend als eine Mischform aus religiöser Gnade und irdischer Schuld, die von einer nicht getilgten Erbschuld wie auch von weltlichen Verbrechen ausgeht. Amnestie ist bei ihm zudem ein kollektives Phänomen, welche die Eintracht in der Gemeinschaft bzw. Synthese von Volk und Intellektuellen im Sinne der počvenniki zum Ziel hat. In seinem letzten Werk Brat'ja Karamazovy zeigt sich Amnestie schließlich innerhalb eines Rituals zur Versöhnung der Gemeinde und als religiös motivierte Verheißung auf das Jenseits.

In Anlehnung an Platon und Aristoteles, dass Strafe, als Heilung durch das Entgegengesetzte wirkt', so ließe sich auch für Dostoevskijs Darstellungen von Amnestien behaupten, dass Heilung im Sinne einer Versöhnung durch Amnestie aus der Darstellung von Schuld und Bekenntnis der Schuld erfolgt. Mit Hilfe der Katharsis oder der Abschreckung kann der Leser selbst von den Untaten erfahren und mündig urteilen, oder wie es Sylvia Sasse in Bezug auf dramaturgische Inszenierungen ausdrückt: „Urteil und Affekt sind Teil der ästhetischen 
Erfahrung. (...) Platon und Aristoteles verstanden dabei Strafe als "Heilung durch das Entgegengesetzte', die vorbeugend und abschreckend wirken soll." (Sasse 124) Der Nachvollzug von Dostoevskijs Texten durch den Leser, die ,Identifikation mit dem Gesehenem', wirkt in Dostoevskijs ,Volkstheater' in jedem Fall kathartisch (123). Doch „Kunst als Strafe“ (ebd.), wie es Sasse vor allem im Hinblick auf die Schauprozesse der Sowjetzeit betrachtet, rückt in Dostoevskijs Texten in den Hintergrund zugunsten eines religiös aufgeladenen Schuldbegriffes.

Schuldbewusstsein kann durch Kunst inszeniert werden, aus dem bei Dostoevskij die Amnestie als kollektive Stimme des Volkes - vox populi - zum Aufruf nach einem besseren Leben und Versöhnung erklingt. ${ }^{10}$ Oder wie es der Rechtsphilosoph Vladimir Solov'ev anlässlich der zweiten Rede zum Andenken an den Tod Dostoevskijs ausdrückte, sei die wichtigste Fähigkeit des russischen Volkes, auf die der Autor hingewiesen hätte:

das Bewusstsein der eigenen Sündhaftigkeit, die Unfähigkeit, seine Unvollkommenheit zu einem Gesetz zu machen und als Recht zu erklären und sich mit ihr zufrieden zu geben; von dort kommt auch die Forderung nach einem besseren Leben, der Durst nach Reinigung und sittlicher Tat. (Solowjew 1992, 34)

Auch wenn Solov'evs Formulierungen altmodisch klingen, seine Forderung nach besserem Leben als Plädoyer für die Selbstbestimmung der russischen Bevölkerung ist aktueller denn je. Es bleibt die Frage, inwiefern Amnestien heutzutage eine positive Wirkung entfalten können, oder inwiefern ein Missbrauch politischer Amnestien zunimmt. Bezogen auf die aktuellen Prozesse um den Oppositionellen Aleksej Naval'nyj bleibt, mit Chodorkovskijs Worten gesprochen, daher ebenso nur das ,Hoffen auf ein Wunder' (a.a.O.).

\footnotetext{
${ }^{10}$ Diese Stimme des Volkes zeigt sich auch an einem anderen Beispiel aus den Brat'ja Karamazowy mit der Figur Dmitrij Karamazov. Mit ihm bescheibt Dostoevskij den Konflikt zwischen Revolutionären und Gottgläubigen, zwischen sozialer und christlicher Ethik, die sich in ein und derselben Person vereinen. Dmitrijs Annahme seiner Schuld am Ende der Brat'ja Karamazovy im Sinne des ethischen Leitsatzes, jeder ist verantwortlich für alles' versucht sich von dem atheistischen Leitsatz ,Alles ist erlaubt' zu lösen, um damit allen Beteiligten ihre Mitschuld vor Augen zu führen. Solov'ev wird diese ethische Gradwanderung schließlich unter dem Begriff der „всечеловечество“ (All-Menschheit) für Dostoevskij postulieren.
} 


\section{Literatur}

Burkhart, Dagmar. „Der ethische Diskurs bei Dostojewskij“. In: Burmeister H. (Hg.) Literatur und Prophetie. Dostojewskijs Blick auf Europa. Rehburg-Loccum: Evangelische Akademie, 2003. 47-66.

Chaniotis, Angelos. „Normen stärker als Emotionen? Der kulturhistorische Kontext der griechischen Amnestie“. In: HarterUibopuu K./Mitthof F. (Hrsg.) Vergeben und Vergessen? Amnestie in der Antike Beiträge zum 1. Wiener Kolloquium zur Antiken Rechtsgeschichte 27.-28.10.2008. Wien: Holzhausen, 2013, 47-70.

Chodorkowski, Michail. „Amnestie“. In: Meine Mitgefangenen. Berlin: Galiani Verlag, 2014. 90-92.

Dostoevskij, Fëdor M. Polnoe sobranie sočinenij v 30-ti tomach [PSS]. Leningrad: Nauka, 1972-1983.

Dostojewski, Fjodor M. Tagebuch eines Schriftstellers. Notierte Gedanken. München: Zweitausendeins, 1992.

Dostojewskij, Fjodor M. Die Brüder Karamasow. 3. Aufl., Frankfurt a.M.: Fischer, 2011.

- „Aufzeichnungen aus dem Totenhaus". In: Schmidt H. (Hg.) Gesammelte Werke in zwanzig Bänden. Berlin/Weimar: AufbauVerlag, 1983.

Drecoll, Volker H. (Hg.) Augustin Handbuch. Tübingen: Mohr Siebeck, 2007.

Frank, Joseph. The mantle of the prophet. 1871-1881. Princton: University Press, 2002.

- Dostoevsky. The Stir of Liberation. 1860-1865. Princeton: University Press, 1986.

Gerigk, Horst-Jürgen. „Versteckte Botschaft: Erzähltechnik als „Mäeutik“ in ,Verbrechen und Strafe“". In: Ders. Ein Meister aus Russland. Beziehungsfelder der Wirkung Dostojewskijs. Vierzehn Essays. Heidelberg: Universitätsverlag Winter $\mathrm{GmbH}$ Heidelberg, 2010. 129-134.

Gerigk, Horst-Jürgen/Neuhäuser, Rudolf. Dostoevskij im Kreuzverhör. Ein Klassiker der Weltliteratur oder Ideologe des neuen Rußland? Zwei Abhandlungen. Heidelberg: Mattes-Verlag, 2008.

Neuhäuser, Rudolf. „Dostojewskij. Schuld und Sühne“. In: Zelinsky B. (Hg.) Der russische Roman. Düsseldorf: Bagel, 1979. 161-188.

Rosenshield, Gary. Western law, russian justice. Dostoevsy, the Jury Trial, and the Law. 2005. Madison, Wisconsin: University of Wisconsin Press.

Sasse, Sylvia. „Gerichtsspiele. Fiktive Schuld und reale Strafe im Theater und vor Gericht“. In: Koch G./Schwarte L./Sasse S. (Hrsg.) Kunst als 
Strafe. Zur Ästhetik der Disziplinierungen. München: Wilhelm Fink, 2003. 123-147.

Solowjew, Wladimir. Reden über Dostojewskij. Hg. v. L. Müller. München: Erich Wewel Verlag, 1992.

Vasmer, Max. „Amnestie“. In: Russisches Etymologisches Wörterbuch. Bd.1 Heidelberg: Winter, 1976. 17.

Zink, Andrea „,Die Arrestanten waren die reinsten Kinder"“ - Zur Rechtfertigung des Verbrechens in Dostoevskijs Aufzeichnungen aus dem Totenhaus". Deutsche Dostoevskij-Gesellschaft. 7, 2000. 115-134.

\section{AMNESTY IN DOSTOEVSKY}

The term amnesty means the waiving of punishment (to a group of people) without, however, erasing the guilt. Amnesties are usually granted in connection with political events such as national or international peace amnesties in times of political change or wars. However, there are also waivers of criminal prosecutions, popularly known as "mercy before justice". Amnesties can therefore be seen, on the one hand, as a humane act of mercy, but on the other hand they can also cause problems in the population's sense of justice, since punishments are treated differently, and the principle of equality is shaken. The concepts of punishment, guilt and innocence, as well as their representation, offer a basis for the question of how amnesties are formed in Dostoevsky's texts.

Dostoevsky's characters are usually ambivalent and challenge a reflective reading as well as an ethical judgement. By making social injustice an important theme in his texts, the author focuses on the restoration of justice through amnesty. A co-responsibility in society as well as a co-guilt in a higher sense form the core of his argumentation, both as a contrast to justice, which considers the guilt of the individual, and as a consideration of man's hereditary guilt and his responsibility to the community. A comparison with ancient Greek jurisprudence also shows that amnesties were closely connected with the collective, with emotion and ritual. This results in new ways of looking at prominent texts by Dostoevsky, as will be shown with the example of The Brothers Karamazov and other works. This will provide a brief overview of how Dostoevsky understands guilt and innocence, what function punishment has in his texts and how amnesty emerges as a result. 
Keywords: amnesty, complicity, innocence, guilt, punishment, ancient jurisprudence, justice, grace 